\title{
Experimental Research and Analysis on a Vibration Model of Stator of L1B2 Linear Ultrasonic Motor
}

\author{
Xingyu Yi, Gang Zhao and Wenlei Xiao \\ School of Mechanical Engineering \& Automation, Beihang University, China \\ ${ }^{*}$ Corresponding author
}

\begin{abstract}
The vibration of stator is an important part of the linear ultrasonic motor operating mechanism. Linear ultrasonic motor is driven by the interface force between stator and slider. Changes in vibration mode are not considered in the traditional motor vibration model which fails to reflect the vibration amplitude variations of LUSM during the process of running. In order to study the vibration of the ultrasonic motor stator, adequate experimental data of vibration of stator and the speed of the slider is necessary. This paper simply introduces the experimental system. According to the experimental results and its analysis, we propose a stator vibration model with variable vibration mode. The experiment is performed to verify the feasibility of this stator vibration model. The simulation results are validated by the measured vibration amplitude data. The paper points out some problems to be improved in future.
\end{abstract}

Keywords_linear ultrasonic motor; vibration model

\section{INTRODUCTION}

Linear ultrasonic motor (LUSM) has been of interest in high precision motion control due to its unique characteristics such as high torque at low speed, fast response, accurate positioning, simple design, compact size, light weight, auto-locking on power, low electromagnetic interferences[1]. Many engineers are focusing on the research and development of LUSM since 1980s. The LUSM has been applied in high precision motion stage and other industrial fields. The piezoelectric stator of the motor is excited to produce bending and longitudinal vibrations, the two orthogonal vibrations generate an elliptical motion on the material points of the driving foot of stator. The slider is in contact with the driving wave crests by the preload, the dynamic contact force produce the linear motion. The piezoelectric linear ultrasonic motor is an electromechanical coupling system. It has two energy conversion stages [2]. In the first stage, the electrical energy converts into ultrasonic frequency mechanical oscillations. In the second stage, depending on the contact force between driving foot and slider the high-frequency vibration is rectified into macroscopic linear motion.

In order to predict the linear ultrasonic motor behavior, numerous of dynamic models of linear ultrasonic motor are proposed to predict the transient and steady-state responses of the LUSM [3, 4], but can't reflect the vibration amplitude variations phenomenon of the experiments. This paper a stator vibration model is proposed, it is found that the model can predict the vibration amplitude in a range of drive voltage.

\section{DESCRIPTION OF THE LUSM INVESTIGATED}

The LUSM investigated in this paper is a double-mode L1B2 ultrasonic motor, the schematic of the motor is illustrated in Figure 1 (a). The structure of the motor consists of the stator with driving foot, a slider and a preload spring. The motor shell clamps the stator in the vibration nodes. The LUSM is driven by the asymmetry of the piezoelectric stator of the motor applying to the electrodes $1-4$ or $2-3$ at a time to produce the first longitudinal mode and the second bending mode to produce the elliptical motion in different directions [5].

\section{THE EXPERIMENT STUDY}

\section{A. Implement of Experimental System}

The experimental system includes the LUSM motion platform, vibration measurement platform, motion control and drive platform. In order to get the vibration of stator in a running process, we design a special LUSM experimental motion platform, which opens a window on the measuring side of LUSM motor. So the laser Doppler vibrometer can measure the vibration. The vibration experiment based on the laser doppler vibrometer, we build a vibration measurement platform consists of the Polytec OFV laser doppler vibrometer, NI-DAQ device and DAQmx. In order to ensure the motion and vibration data of LUSM is accurate and synchronous, a 50nm linear grid ruler is used in the motion platform and a special EtherCAT driving controller is designed. The linear grid ruler makes sure the experiment can obtain the accurate location and speed data of slider. The EtherCAT driving controller which uses LC resonance voltage step-up circuit to provide the high-voltage at the operating frequency uses the EtherCAT as the motion data and control signal exchange protocol between the host computer and the driving controller. The host computer uses TwinCAT a real time automation PC controller with a multi-PLC system. The system architecture is illustrated in Figure I (B). 


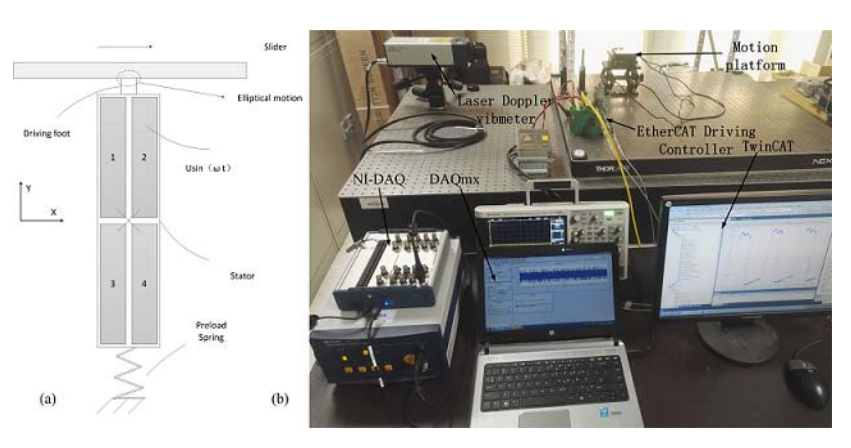

FIGURE I. LUSM SCHEMATIC (A) AND SYSTEM ARCHITECTURE (B)

\section{B. Speed Experimental Results and Analysis}

Figure II (A) shows the speed performance of the slider. The ideal steady-state speed of the slider in response to a step control signal is steady. But in the actual operation of the linear ultrasonic motor, the speed is not stable. In this paper, we adjust the voltage amplitude with the special ultrasonic frequency to adjust the speed of LUSM. Figure II (B) shows the relationship between speed in a special voltage and position of the slider in three experiments. In other speed experiments, the same phenomenon appears. The speed of the slider is changing during the process of running and the value of speed is related to the position where the slider is in contact with the stator.

\section{Vibration Experimental Results and Analysis}

The elliptical motion of the driving foot of the stator is the important key of linear ultrasonic motor motion. The elliptical directly decides the output performance of linear ultrasonic motor. Figure II (C) shows the relationship between vibration amplitude in the same voltage and position of the slider in three experiments. In other vibration experiments, the same phenomenon appears. The vibration amplitude of stator is changing during the process of running. The vibration experimental results show that the linear ultrasonic motor's vibration amplitude will be not stable after start up and the vibration amplitude is related to the position where the slider is in contact with the stator.
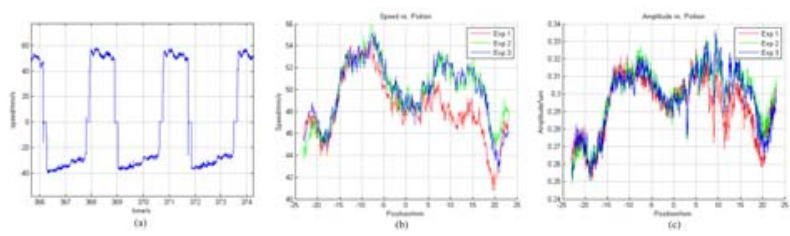

FIGURE II. SPEED PERFORMANCE (A), RELATIONSHIP BETWEEN SPEED IN A SPECIAL VOLTAGE AND POSITION (B) AND RELATIONSHIP BETWEEN VIBRATION AMPLITUDE AND POSITION (C)

\section{Analyses of Speed and Vibration Experimental Results}

The speed and vibration experimental results show that the relationship between the speed of the slider and the vibration amplitude of stator is proportional. That means the essence of LUSM speed adjustment is to adjust the vibration amplitude of the driving foot of the stator. A reasonable explanation the unstable of the speed is that the vibration amplitude of the stator is changing during the process of running.

\section{THE VIBRATION MODEL}

\section{A. Analytical Model}

According to the operating mechanism of LUSM, a vibration model of LUSM is derived. The bending vibration of the stator is treated as the bending of a beam [1]. Applying the method of separation of variables, the lateral vibration displacement is expressed as follows

$$
w(x, t)=\phi(x) q(t) .
$$

The function $\mathrm{q}(\mathrm{t})$ indicates harmonic motion, and the function $\phi(\mathrm{x})$ represents $\mathrm{B} 2$-mode shape. According to vibration experimental phenomenon that the bending vibration amplitude changed and is related to the position where the stator contact with the slider, the constant the free-free boundary B2-mode is not suitable. The function $\phi(x)$ considering the effect of axial force $\mathrm{P}$ on the bending vibration mode with a free-free boundary is written as

$$
\begin{gathered}
\phi(x)=\cos \beta_{1} x+D \sin \beta_{1} x+E \sinh \beta_{2} x+F \cosh \beta_{2} x \\
\beta_{1}{ }^{2}=\left[(P /(2 E I))^{2}+\rho S /(E I) \omega^{2}\right]-P /(2 E I) \\
\beta_{2}{ }^{2}=\left[(P /(2 E I))^{2}+\rho S /(E I) \omega^{2}\right]+P /(2 E I)
\end{gathered}
$$

According to the free-free boundary[1], Eq. 5, Eq. 6, Eq. 7 and Eq. 8 can be derived.

$$
\begin{aligned}
& -\beta_{1}^{2}+\mathrm{F} \beta_{2}^{2}=0 \\
& -\mathrm{D} \beta_{1}^{2}+\mathrm{E} \beta_{2}^{2}=0 .
\end{aligned}
$$

$-\beta_{1}{ }^{2} \cos \beta_{1} 1-D \beta_{1}^{2} \sin \beta_{1} 1+E \beta_{2}^{2} \sinh \beta_{2} 1+F \beta_{2}^{2} \cosh \beta_{2} 1=0$

$$
\beta_{1}^{2} \cos \beta_{1} 1-D \beta_{1}^{2} \sin \beta_{1} 1+E \beta_{2}^{2} \sinh \beta_{2} 1+F \beta_{2}^{2} \cosh \beta_{2} 1=0
$$

The 1, S, E, $\rho$ and $\omega$ are the length of the stator, the cross section area, elastic modulus of material, mass density and frequency of vibration, respectively. According to the energy method and Lagrange equation, the bending mode of the stator can be obtained $[1,6]$.

$$
\mathrm{Mq}^{\prime \prime}(\mathrm{t})+\mathrm{Cq}^{\prime}(\mathrm{t})+\mathrm{Kq}(\mathrm{t})=\mathrm{A}_{\mathrm{v}} \mathrm{V}_{0} \sin \left(\mathrm{w}_{\mathrm{d}} \mathrm{t}\right)+\mathrm{Ft}(\phi(0)-\phi(1))
$$

The M, C and $\mathrm{K}$ are the mass, damping and stiffness for the B2-mode of stator, respectively. V0 and $\omega \mathrm{d}$ are the amplitude of the driving voltage and the driving frequency, respectively. Av is the force factor related to the electromechanical coupling effects. Subjected to the excitation of the piezoelectric stator, 
the steady-state bending vibration response of the beam can be expressed as

$$
\begin{gathered}
\mathrm{w}(\mathrm{x}, \mathrm{t})=\mathrm{F}_{\mathrm{d}} \phi(\mathrm{x}) / \mathrm{K}\left[\left(1-\left(\omega_{\mathrm{d}} / \omega_{\mathrm{n}}\right)^{2}\right)^{2}+\left(2 \mathrm{C} /(2 \mathrm{MK})\left(\omega_{\mathrm{d}} / \omega_{\mathrm{n}}\right)\right)^{2}\right]^{-1 / 2} \\
\sin \left(\mathrm{w}_{\mathrm{d}} \mathrm{t}-\varphi\right) .
\end{gathered}
$$

This model focus on the steady-state of the vibration, the time-varying force $\mathrm{Ft}$ is simplified to constant generalized effect. The tangential force Ft is related to the force $\mathrm{P}$. The force factor is $A_{p}$, so the effect of the generalized force simplified to

$$
\mathrm{Fd}=\mathrm{A}_{\mathrm{v}} \mathrm{V}_{0}+\mathrm{A}_{\mathrm{p}} \mathrm{P}
$$

\section{B. Identification and Simulation}

According to the experimental results, it is obvious that the bending vibration amplitude is related to the position where the stator contact with the slider, so that the axial force $\mathrm{P}$ which affects the B2-mode is related to the position. The staedy-state vibration amplitude is the function of position. The vibration experimental results reflect the relationship between vibration amplitude and position. And we obtain the relationship between the axial force $\mathrm{P}$ and vibration amplitude by the analytical model, so we can identify the relationship between the axial force $\mathrm{P}$ and position. Some parameter used for simulation list in Table I, the rest of the parameters are drove according to the energy method and Lagrange equation, and the driving voltage is obtained from experiments.

According to the identification of the axial force P. The identification results is shown in Figure III (a) and (b). It is found that the identification results can be divided into three parts according to the driving voltage: low voltage zone, medium voltage zone and high voltage zone.In low voltage zone $(0 \mathrm{~V} \sim 353 \mathrm{~V})$, the vibration amplitude is small, some axial force $\mathrm{P}$ can't be identified. In medium voltage zone $(361 \mathrm{~V} \sim 410 \mathrm{~V})$, there is no uniform rule in the identification results. In high voltage zone $(450 \mathrm{~V} \sim 675 \mathrm{~V})$, there are similar rules in the identification results.
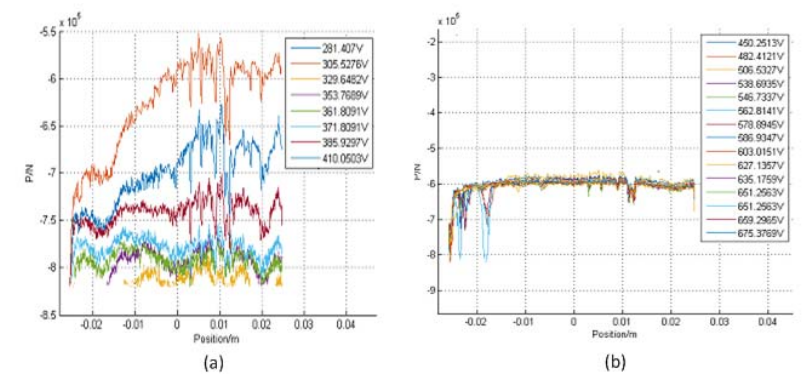

FIGURE III. IDENTIFICATION RESULTS IN LOW AND MEDIUM VOLTAGE ZONE (A) AND IDENTIFICATION RESULTS IN HIGH VOLTAGE ZONE (B)

According to the identification results, a stator vibration model with variable vibration mode suitable for the high voltage zone is proposed. The simulation results are shown in
Figure IV (a), and the relative error of the model is shown in Figure IV (b). The stator vibration model applied in the high voltage zone can predict the vibration amplitude for every position where the stator contacts with the slider effectively.
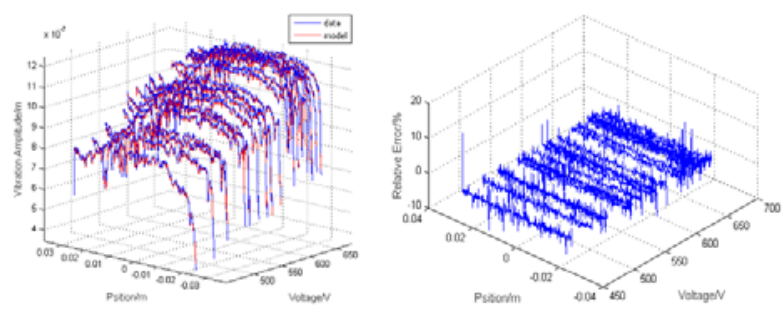

(a)

(b)

FIGURE IV. SIMULATION RESULTS OF THE VIBRATION MODEL

TABLE I. PARAMETERS

\begin{tabular}{|c|c|c|c|}
\hline Parameter & Value & $\mathrm{S}$ & $2.25 \mathrm{e}-5 \mathrm{~m}^{2}$ \\
\hline 1 & $0.03 \mathrm{~m}$ & $\mathrm{C}$ & $178 \mathrm{Ns} / \mathrm{m}$ \\
\hline$\omega_{\mathrm{d}}$ & $3.0473 \mathrm{e}+05 \mathrm{rad} / \mathrm{s}$ & $\mathrm{I}$ & $1.0544 \mathrm{e}-10 \mathrm{~m}^{4}$ \\
\hline $\mathrm{E}$ & $8.6957 \mathrm{e} 10 \mathrm{~N} / \mathrm{m}^{2}$ & $\mathrm{c}_{66}{ }^{\mathrm{E}}$ & $3 \mathrm{e} 10 \mathrm{~N} / \mathrm{m}^{2}$ \\
\hline $\mathrm{c}_{11}{ }^{\mathrm{E}}$ & $5.1 \mathrm{e} 10 \mathrm{~N} / \mathrm{m}^{2}$ & $\mathrm{e}_{31}$ & $24.8 \mathrm{cl} / \mathrm{m}^{2}$ \\
\hline
\end{tabular}

V SUMMARY

In this paper, a stator vibration model of LUSM is proposed to predict the stator vibration amplitude. This model differs from the previous reported model, the effect of axial force on the bending vibration mode is considered in the operation of the linear ultrasonic motor. The vibration experiments are designed to examine the feasibility and effectiveness of the proposed model. It is shown that the axial force in different position makes a significant impact on the bending vibration of the stator, which directly influence on the speed of the slider. In future research, the dynamic axial force and tangential force will be considered to make the model can be used for low and medium voltage zone.

\section{ACKNOWLEDGEMENT}

The authors would like to acknowledge the support of National Natural Science Foundation of China (No.51505020).

\section{REFERENCES}

[1] Zhao, Ultrasonic Motor Technologies and Applications, Science Press, Beijing, 2007.

[2] S. Ueha, Y. Tomikawa, Ultrasonic Motors: Theory and Applications, Clarendon Press, Oxford, 1993.

[3] X. Li, Z. Yao, R. Wu, Modeling and analysis of stick-slip motion in a linear piezoelectric ultrasonic motor considering ultrasonic oscillation effect, J. International Journal of Mechanical Sciences 107(2016)215-224.

[4] X. Li, Z. Yao, Q. Lv, Z. Liu, Modeling stick-slip-separation dynamics in a bimodal standing wave ultrasonic motor, J. Journal of Sound \& Vibration 382(2016)140-157.

[5] G. Peled, R. Yasinov, N. Karasikov, Performance and Applications of L1B2 Ultrasonic Motors, J. Actuators 5.2(2016) 15.

[6] M. S. Tsai, C. H. Lee, S. H. Hwang, Dynamic modeling and analysis of a bimodal ultrasonic motor, J. IEEE Transactions on Ultrasonics Ferroelectrics \& Frequency Control 50.3(2003) 245. 